\title{
Initial experience with magnetic resonance imaging-safe pacemakers
}

\author{
A review
}

\author{
Werner Jung • Vlada Zvereva • Bajram Hajredini • \\ Sebastian Jäckle
}

Received: 6 February 2011 / Accepted: 28 July 2011 / Published online: 13 October 2011

(C) The Author(s) 2011. This article is published with open access at Springerlink.com

\begin{abstract}
Due of its superior soft tissue imaging capabilities, magnetic resonance imaging (MRI) has become the imaging modality of choice in many clinical situations, as illustrated by the tremendous growth in the number of MRIs performed over the past 2 decades. In parallel, the number of patients who require pacemakers or implantable cardiac defibrillators is increasing as indications for these devices broaden and the population ages. Taken together, these phenomena present an important clinical issue, as MR scans are generally contraindicated - except in urgent situationsin patients who have implanted cardiovascular devices. Potentially deleterious interactions between the magnetic fields and radio frequency (RF) energy produced by MR equipment and implantable devices have been identified, including inhibition of pacing, asynchronous/high-rate pacing, lead tip heating, and loss of capture. New devices that incorporate technologies to improve MR safety in patients with pacemakers have recently received approval in Europe and are under evaluation in the United States. Initial data from these devices suggest that these devices are safe in the MRI environment.
\end{abstract}

Keywords Magnetic resonance imaging - MRI · Pacemakers · Implantable cardiac defibrillators

\footnotetext{
W. Jung $(\bowtie) \cdot$ V. Zvereva $\cdot$ B. Hajredini $\cdot$ S. Jäckle Department of Cardiology, Academic Teaching Hospital of the University of Freiburg, Schwarzwald-Baar Klinikum, Vöhrenbacher Street 23,

78050 Villingen-Schwenningen, Germany

e-mail: werner.jung@sbk-vs.de
}

\section{Introduction}

Increasingly, magnetic resonance imaging (MRI) has become the imaging modality of choice across a broad range of indications. It provides superior soft tissue contrast, is not obstructed by bone, and provides a three-dimensional image of anatomical structure that is valuable in guiding both diagnosis and treatment without exposing patients to the risks associated with ionizing radiation, invasive procedures, or iodinated contrast agents. For these reasons, MRI is the fastest-growing imaging modality and has become the standard of care for the evaluation of disease and treatment management across multiple therapeutic areas, includingbut not limited to - oncology, central nervous system and musculoskeletal disorders, and, to an increasing extent, cardiovascular disease. Based on United States data from 1993 to 2002, MRI procedures increased dramatically from 7.7 million to nearly 22 million [1]; the most recent data suggest that there were 27.5 million procedures in 2007 (Fig. 1) [2]. Worldwide, approximately 60 million scans are performed each year [3].

Parallel to the growth of MRI as the imaging modality of choice in many clinical situations, the number of patients with implantable cardiac devices, such as pacemakers and implantable cardiac defibrillators (ICDs), has been increasing steadily as the population ages and indications and recommendations for these devices expand. The rapid growth in MRI and the increase in patients who require implantable cardiac devices have led to a situation in which $50 \%$ to $75 \%$ of patients with implanted devices will be indicated for MRI scans over the lifetime of their device [1].One study found that during a 12month follow-up period, $17 \%$ of patients with pacemakers required a diagnostic MRI [4].

Pacemakers and ICDs contain metal with variable ferromagnetic qualities, complex electrical systems, and $\geq 1$ leads 
Fig. 1 Total MRI procedure volume in millions, 2000-2007, hospital and nonhospital sites [2]. Reprinted with permission from AuntMinnie.com
Total MRI procedure volume in millions,

2000-2007, hospital and nonhospital sites

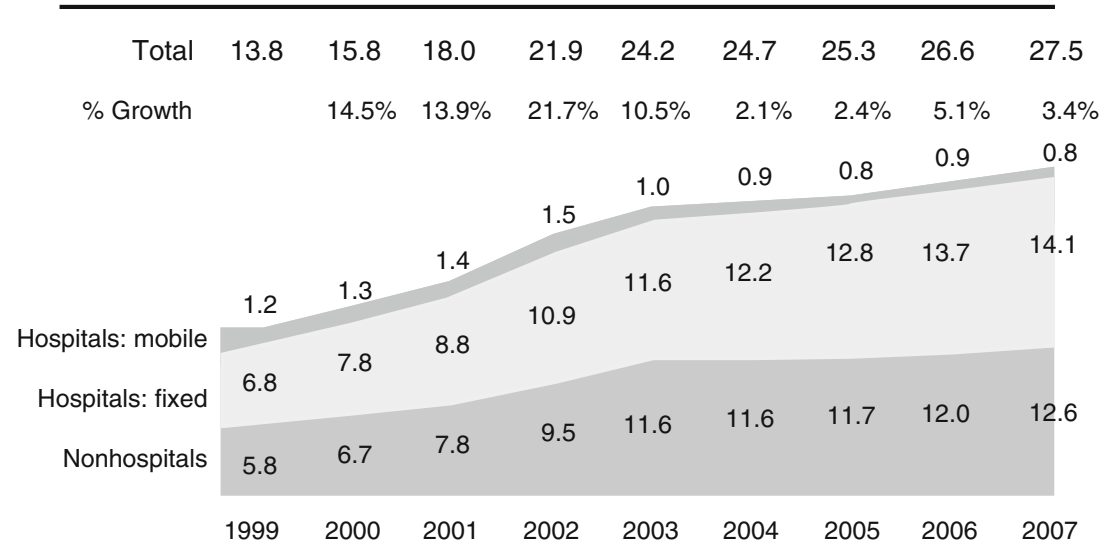

implanted in the myocardium. The static main magnetic field, radio frequency (RF) energy, and gradient magnetic fields associated with MRI have the potential to interfere with pacemaker function. For this reason, significant controversy exists regarding the safety of MRI examination in patients with implantable cardiac devices, particularly in light of the increasing field strengths of clinical MRI scanners. In general, the American Heart Association guidelines indicate that MRI examination of nonpacemaker-dependent patients is discouraged, except in cases with a strong clinical indication and in which the benefits clearly outweigh the risks [5]. Among pacemaker-dependent patients and those with ICDs, MRI examination is generally not recommended except under highly compelling circumstances [5]. As a result of these concerns, no implantable cardiovascular electronic devices have United States Food and Drug Administration (US FDA) approval or Centers for Medicare and Medicaid Services coverage for use in the MRI environment, and "Do not use MR imaging for patients who have an implanted device" appears on product labels. Support for this contraindication came from several reports of lethal consequences associated with MRI in patients with implanted pacemakers [6-8]. This creates a significant problem in that annually approximately 200,000 patients with devices may be denied a scan due to the presence of an ICD [1,9].

In light of the current controversy regarding the safety of MRI scans in patients with pacemakers or ICDs, this review focuses first on an evaluation of the potential risks of scanning in this patient population, followed by an examination of new technologies intended to reduce or eliminate these concerns.

\section{Risks of MRI in patients with pacemakers}

There are a number of potential interactions between MRI and components of pacing and defibrillation systems, including the device, the lead (including abandoned leads), and the system itself (Table 1) [1]. Variables affecting the magnitude of risk are summarized in Table 2.

Static magnetic fields exert mechanical forces on ferromagnetic components within the device [10], which may rotate, dislodge, or accelerate ferromagnetic devices toward the magnet, potentially resulting in serious patient harm. Furthermore, static magnetic fields may result in unpredictable magnetic sensor activation and reed-switch closure, potentially resulting in reversion to asynchronous pacing when the switch is closed or falsely classifying electromagnetic interference as cardiac activity and inhibit-

Table 1 Potential effects of magnetic resonance imaging on pacemakers [10]

Potential effects of MRI on pacemakers

(1) Static magnetic field

Mechanical forces on ferromagnetic components

Unpredictable magnetic sensor activation, reed-switch closure

Changes in electrocardiograms

(2) Modulated radio frequency field

Heating of cardiac tissue adjacent to lead electrodes

Possible induction of life-threatening arrhythmias (very rare)

Pacemaker reprogramming or reset

RF interactions with the device (over- and undersensing)

(3) Gradient magnetic field

Possible induction of life-threatening arrhythmias (unlikely in bipolar mode)

Induced voltages on leads cause over- and undersensing

(4) Combined field effects

Alteration of device function because of electromagnetic interference

Mechanical forces (vibration)

Electronic reset of device

Damage to pacemaker/implantable cardiac defibrillator and/or leads 
Table 2 Potential variables affecting the magnitude of risks in pacemaker/ICD patients undergoing MRI

$\begin{array}{ll}\text { (1) } & \text { Pacemaker design } \\ & \text { Ferromagnetic content } \\ & \text { Switch design (reed vs. Hall) } \\ & \text { Availability of dedicated modes for use during MRI } \\ & \text { Lead design and length } \\ \text { (2) } & \text { Lead geometry } \\ & \text { Lead input filtering capacitance } \\ \text { (3) } & \text { Blood flow at lead/tissue interface } \\ \text { (4) } & \text { Presence of abandoned leads } \\ \text { (5) } & \text { MRI scan duration } \\ \text { (6) } & \text { Strength of RF field } \\ \text { (7) } & \text { Isocenter of scan } \\ \text { (8) } & \text { Type of imaging sequence } \\ (9) & \text { Patient and device/lead position within scanner } \\ (10) & \text { Type and extent of patient monitoring }\end{array}$

ing the need for pacing support when the switch is open [10]. RF interactions with the device may result in oversensing (resulting in high-rate pacing beyond the pacemaker rate limit), undersensing, cardiac tissue heating and thermal damage, pacemaker reset, and, in rare cases, induction of life-threatening arrhythmias. Furthermore, rapidly changing gradient magnetic fields have the potential to induce voltages on leads that may cause oversensing and undersensing. Combined field effects may result in component failures, mechanical vibration, or device damage [10]. The main risks to pacemakers in patients undergoing MRI can be placed into three categories: (1) inhibition of pacing, (2) asynchronous/high-rate pacing, and (3) lead tip heating and loss of capture.
The potential effects of 0.5-T MRI on pacemakers were initially evaluated by Lauck and colleagues [11] using a phantom model with seven dual-chamber and two singlechamber systems. Upon entering the static magnetic field, the reed switch was activated followed by asynchronous stimulation. The subsequent scan did not influence the stimulation function or the pacemaker program, and the event counter function remained intact. In pacemakers with automatic mode switching to demand pacing or programmed evaluation of the reed switch, triggering was observed in the dual-chamber mode and inhibition was observed in the single-chamber mode during the scan. Changes in the pacemaker program or rapid pacing were not observed. The authors concluded that pacemakers should be programmed in the asynchronous mode during the scan to avoid inhibition and trigger mechanisms. The effects of 0.5-T MRI were also assessed in 32 patients (34 MRI examinations) with implanted pacemakers, with measurements at baseline, immediately after MRI, and 3 months after MRI [12]. At this field strength, lead impedance and sensing and stimulation thresholds were unaltered by MRI; however, battery current and impedance increased after the scan. Although battery voltage decreased immediately after MRI, 3-month measurements indicated that voltage recovered fully. Notably, temporary deactivation of the reed switch occurred in more than one-third of patients (12 of 32).

The potential for inhibition of pacing is illustrated in Fig. 2 and was demonstrated in a study conducted by Mollerus and colleagues [13]. In this study 52 nonpacemaker-dependent patients with a total of 119 leads underwent 59 MRI scans of any landmark, using standard peak absorption rate settings for the scan. Devices were

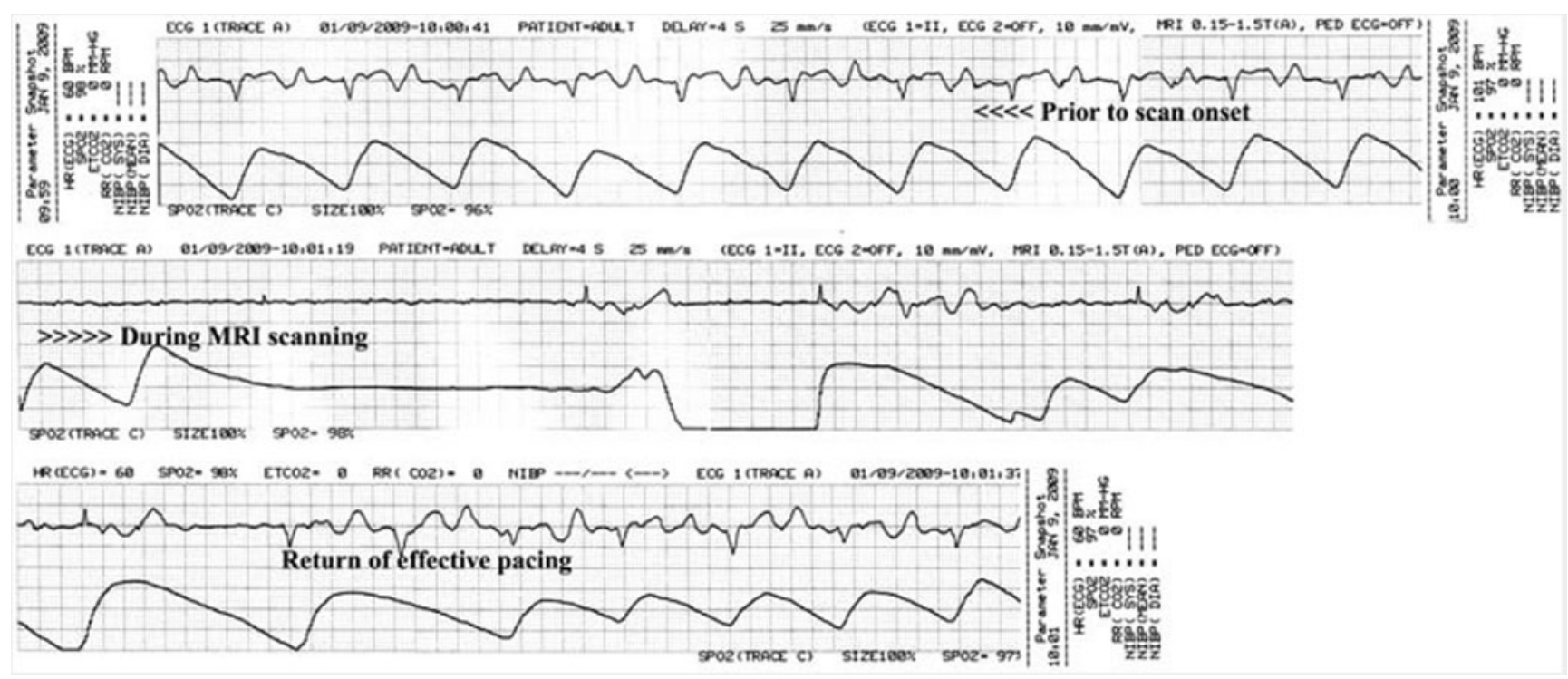

Fig. 2 Potential for inhibition of pacing [26] 
programmed to single-chamber demand mode (VVI) or dual-chamber demand mode (DDI) with a lower rate of $40 \mathrm{bpm}$. Pacing outputs and sensitivities were not changed for the scan, and magnet mode was disabled. If an ICD was present, therapy features were disabled. Telemetry and pulse oximetry plethysmographic waveform were continuously evaluated for ectopy (defined as $\geq 20$ ectopic beats during the entire scan) throughout the scans [13]. A total of seven patients had significant ectopy observed either on telemetry or from observation of the oxygen saturation plethysmographic waveform. No scans were associated with changes in pacing thresholds; however, there were small decreases in sensing amplitudes and pacing impedances [13].

Martin and colleagues [14] reported on a series of 54 nonpacemaker-dependent patients who underwent $62 \mathrm{MR}$ examinations using a 1.5-T MR system. Notably, the type of MRI examination was not limited and included cardiac, vascular, and general MRI studies using various wholebody, averaged, specific absorption rates (SARs); nor was the type of pacemaker present in the patient restricted. Pacemakers were interrogated before and immediately after MRI scanning, and patients were continuously monitored during scanning. Although no adverse events occurred, pacing threshold changes were observed in $40(37 \%)$ of leads, including ten that were judged clinically significant and two that required a change in programmed output. Martin and colleagues [14] conclude that "safety was demonstrated in this series of patients"; however, it is important to note that no patients underwent long-term follow-up, a particular concern in patients who experienced a significant change in pacing thresholds.

Electrical reset (e.g., restoration of the factory setting with predefined synchronous pacing mode and parameters) has been demonstrated to occur in patients with implanted devices subjected to MRI. Reset is an emergency mode that is a safety feature to guarantee at least minimal pacemaker functionality in the event of battery voltage dips. In a prospective study conducted by Sommer and colleagues [15], nonpacemaker-dependent patients with an urgent need for an MRI examination underwent a total of 115 MRI examinations at $1.5-\mathrm{T}$. Pacemakers were reprogrammed prior to MRI, such that if the heart rate was $<60 \mathrm{bpm}$, the asynchronous mode was used to reduce the risk for MRI-induced inhibition; if the patient had a heart rate of $>60 \mathrm{bpm}$, the sense-only mode was programmed to reduce the potential for MRI-induced competitive pacing and potential proarrhythmia. Pacemakers were interrogated before and after the scan and after 3 months, including measurement of the pacing capture threshold and serum troponin I levels [15]. Although all MRI examinations were completed safely, post-MRI interrogation showed that electrical pacemaker reset occurred in seven $(6.1 \%)$ examinations. In all cases, the pacemaker could be reprogrammed to the parameters used prior to the MRI scan [15].

The potential for electrical reset presents a safety hazard for two reasons. First, in cases in which a reset occurs concomitant with an open reed switch, bradycardia/asystole may occur in patients with low intrinsic heart rates as a result of inhibition of pacemaker output by time-varying gradient fields. Second, the default pacing mode may not necessarily provide adequate functionality for certain patients [10].

The reed switches contained in most current pacemakers are susceptible to the magnetic fields generated during MRI. The state of the reed switch in various orientations and positions in the magnetic fields of 0.5. 1.5 , and $3.0 \mathrm{~T}$ was evaluated by Luechinger and colleagues [16]. When oriented parallel to magnetic fields, reed switches closed at $1.0 \pm 0.2 \mathrm{mT}$ and opened at $0.7 \pm 0.2 \mathrm{mT}$. In low magnetic fields $(<50 \mathrm{mT})$ reed switches were closed, and in high magnetic fields $(>200 \mathrm{mT})$ reed switches opened in $50 \%$ of orientations [16]. These data suggest that the position of the reed switch may not be predictable in patients undergoing MRI, a hypothesis supported by the fact that the reed switch remained inactivated in $44.7 \%$ of patients in the Sommer et al. study [15].

Heating results from power deposition by the RF radiation used in MRI and is dependent on SAR, position of the pacemaker lead loop in the RF coil, configuration of lead, and the lead model [15, 17]. RF-induced tissue damage is also a function of both temperature and exposure time. The electrode-tissue boundary is particularly vulnerable to heating, resulting in a potential risk for thermal injury of the tissues adjacent to the electrode, deterioration of the pacing thresholds, and atrial or ventricular perforation. In vitro, temperature increases of up to $63.1^{\circ} \mathrm{C}$ have been seen at $1.5 \mathrm{~T}$ [18]; animal studies have demonstrated increases of up to $20.4^{\circ} \mathrm{C}$ at $1.5 \mathrm{~T}$ and an SAR of $3.8 \mathrm{~W} / \mathrm{kg}$ [19]. Such changes in temperature have the potential to cause serious harm to the patient as a result of loss of pacemaker capture [20]. Pacemaker leads abandoned after pulse generator removal present similar problems, regardless of whether they are capped or otherwise electrically intact [10].

In the study conducted by Sommer and colleagues [15] (summarized previously), cardiac troponins were increased in four of 114 examinations; in one case these increases were associated with a significant increase in pacing capture threshold. Moreover, there were six pacing capture threshold changes $\geq 1.0 \mathrm{~V}$ in 195 leads, and statistical analysis showed that there was a significant increase in pacing capture threshold from pre- to post-MRI. None of these changes had clinically relevant effects (e.g., requiring an increase in pacemaker output to ensure stimulation within a sufficient safety margin) [15]. Of note, possible long-term effects of scanning were identified, in that two 
cases of increased pacing capture threshold were not detected until the 3-month follow-up [15]. The authors speculate that scar tissue development around the lead tips, as a result of RF-related thermal injury, was the cause of these changes. Notably, this study was conducted with significant precautions against RF-induced heating, including limiting the SAR to $<1.5 \mathrm{~W} / \mathrm{kg}$ and excluding anatomic regions with full coverage of the pacemaker lead loop, including the thoracic spine, heart, and breasts. The total active scan time was also limited to $30 \mathrm{~min}$ [15].

The results from the Sommer and Martin studies are at odds with an earlier, small (40 patients) series published by Mollerus and colleagues [21], in which no cases of troponin elevations or changes in capture thresholds were observed. It remains open to debate whether MRI scans will produce sufficient tissue heating to cause enough myocardial cell necrosis to result in cardiac biomarker release.

\section{MRI-safe devices: features}

As a result of the risks imposed by MRI in patients with pacemakers and ICDs - and the increasing importance of MRI as a diagnostic tool-considerable research has been conducted to develop devices that are safe in this environment. To improve the safety of these devices, components have been redesigned and new features, particularly lead design and geometry, have been incorporated into these systems.

Multiple features are required to improve the safety of pacemakers and ICDs in the MRI environment, including improved control of reed switch behavior, better protection against electromagnetic interference leading to electrical reset, dedicated MRI modes for specific patient populations, andperhaps most importantly - pacemaker leads with no or minor RF-induced temperature increases. The SureScan ${ }^{\mathrm{TM}}$ pacing system, which is approved in Europe and is under evaluation in the United States, has incorporated many of these features to improve safety during an MRI scan. These features include changing the lead input-filtering capacitance to minimize the energy induced on leads/discharged at the tip, replacement of the reed switch with a Hall sensor, internal power supply circuit protection, and a reduction in ferromagnetic components (Table 3). The device provides dedicated modes with clear steps to provide predictable pacemaker performance during the scan and includes radiographic markers to improve device identification.

\section{MRI-safe devices: testing and clinical experience}

At present, two systems "MRI-conditional" devices are available, the Revo system (Medtronic) and the ProMRI system (Biotronik). It is important to note that these devices, although specifically designed for the MRI environment, have their own set of limitations. For example, the Revo system limits the static magnetic field strength to $1.5 \mathrm{~T}$, and the patient must be positioned within the bore such that the isocenter is superior to the $\mathrm{C} 1$ vertebra and inferior to the T12 vertebra. The whole-body averaged SAR must be $\leq 2.0 \mathrm{~W} / \mathrm{kg}$, and the head SAR must be $<3.2 \mathrm{~W} / \mathrm{kg}$. The ProMRI system requires that a field strength of $1.5 \mathrm{~T}$ not be exceeded and has similar isocenter limitations. Both devices recommend that proper patient monitoring be provided during the MRI scan, including visual and verbal contact with the patient, electrocardiography, and pulse oximetry.

At present, there are no publicly available data on completed or ongoing studies to confirm the MRI safety of the ProMRI system. However, the EnRhythm MRI study evaluated the Revo pacemaker and lead system designed to minimize lead tip heating and provide a programmable MRI pacing mode in a worldwide, prospective, randomized, controlled, unblinded clinical trial [3]. The study enrolled patients with standard indications for a dualchamber pacemaker implantation; successfully implanted patients were randomized in a 2:1 ratio to undergo MRI or to have no MRI scan at 9 to 12 weeks after pacemaker system implantation. At this time patients received 14 clinically relevant head and lumbar scan sequences per-

Table 3 New technologies incorporated in MR-conditional pacing systems

\begin{tabular}{ll}
\hline Change & Potential benefit \\
\hline Lead input filtering capacitance & Minimize energy induced on leads/discharged at tip \\
Reed switch replaced by Hall sensor & Control switch behavior \\
Internal power supply circuit protection & Prevents energy induced on telemetry antenna from disrupting internal power supplies \\
Reduction in ferromagnetic components & Decreases susceptibility to magnetic attraction \\
Dedicated modes for use during MRI & Suspension of diagnostic data collection and atrial arrhythmia therapy \\
Lead geometry changes & Reduce lead heating \\
Radiographic markers & Allows identification of MRI-conditional devices \\
\hline
\end{tabular}


formed on 1.5-T machines, maximizing gradient slew rate and/or transmitted power up to an SAR of $2 \mathrm{~W} / \mathrm{kg}$ (control patients waited $1 \mathrm{~h}$ with no MRI). All patients were evaluated before and 1 week and 1 month after the study visit [3].

An initial report of this study was recently published [22]. At the time of the report, a total of 464 patients had been implanted at multiple centers in the United States, Europe, Canada, and the Middle East. There were no reports of MRIrelated complications or MRI-attributed sustained ventricular arrhythmias, asystole episodes, or pacemaker malfunctions. The system-related, complication-free rate was $91.7 \%(P<$ 0.001). At 1 month after the MRI visit, capture thresholds, sensing amplitudes, and lead impedance were stable and similar between MRI and control patients. In all cases except one control ventricular lead, pacing capture threshold increases were $\leq 0.5 \mathrm{~V}$. Sensing amplitude decreases of $>50 \%$ (or to $<1.5 \mathrm{mV}$ in an atrial lead or $<5.0 \mathrm{mV}$ in a ventricular lead), were observed for atrial leads in $5.3 \%$ and $7.2 \%$ of MRI and control patients, respectively, and for ventricular leads in $3.0 \%$ and. $5.1 \%$ of MRI and control patients, respectively. No evidence was found for clinical (bradycardia or tachycardia), subclinical (pacemaker performance), or technical (pacemaker or lead damage) adverse events observed in patients receiving an MRI.

A second single-center, controlled, nonrandomized study recently reported by Forleo and colleagues [23], provides a direct safety and efficacy comparison between an MRIcompatible pulse generator and lead system with conventional dual-chamber pacing (DDD) implant outcomes. Because the redesign of the system to be MRI-safe required increased lead diameter and stiffness, implantation success was also evaluated in this small study. In the study, 107 consecutive patients were implanted with either the MRIcompatible device or a dual-chamber, active-fixation lead non-MRI system. Data were collected at implant and during postoperative follow-up through 12 months. The implantation success rate in both groups was $100 \%$; the desired values for pacing threshold, $\mathrm{P}$ - and R-wave amplitudes, and impedance were obtained in all patients [23]. As expected, given the increased diameter of MRI-safe leads, successful cephalic vein access of both leads was slightly, though not significantly, lower in patients who received MRI leads (60.0\%) compared with DDD patients (68.4\%). Subclavian vein puncture to place at least one lead was required in $40.0 \%$ of MRI patients and $31.6 \%$ of DDD patients. Overall, there was no difference in procedure times. Fluoroscopy time in the DDD group was shorter; however, this difference did not reach statistical significance. There were no cases of high-threshold or inadequate sensing. Importantly, there was no difference between the two groups in the incidence of complica- tions at implant; no chronic lead displacement, exit block, sensing problem, lead conductor fracture, insulation defect, or infections were observed during the follow-up period. These data-while not indicative of the performance of the system in the MRI environmentindicate that the MRI-compatible system can be used safely and effectively for cardiac pacing.

\section{Conclusions}

Although some studies suggest that with appropriate precautions, MRI scans can be conducted safely in patients with pacemakers or ICDs, it is apparent that MRI scans have the potential to cause serious deleterious effects in this patient population. Moreover, as noted by Gimbel in 2009, only about 1500 scans have been reported on device patients in the medical literature [24], and the vast majority of these reports are from studies conducted at institutions with significant expertise in MRI and electrophysiology [5]. Moreover, it is highly likely that the majority of adverse events occurring during MRI scans of patients with pacemakers or ICDs have gone unreported. These concerns are also highlighted by an editorial, written by Faris and Shein on behalf of the US FDA, that states "while the FDA recognizes that there are pacemaker and ICD patients for whom, on a case-by-case basis, the diagnostic benefit from MRI outweighs the presumed risks, we believe that those risks have not yet been characterized and mitigated sufficiently to justify the routine use of MRI examination in those populations" [25]. It is also worthwhile to note that MRI technology is evolving with higher static magnetic fields, faster gradient fields, and stronger RF fields. As such, the majority of currently published data may not reflect clinical reality in terms of the risks imposed by newer MRI technologies.

The value of MRI in a broad range of patient populations is indisputable and, as noted earlier, the number of patients with indications for such scans is likely to grow significantly in the future-there is already a significant overlap between patients who require pacemakers or ICDs and those who would ideally undergo scanning. The availability of MRI-safe technologies reduces the concerns associated with MRI scanning in patients with pacemakers and ICDs.

Acknowledgement The author acknowledges the editorial assistance of John R Ferguson and Tara Fish, whose services were funded by Medtronic, Inc. The author meets criteria for authorship as recommended by the International Committee of Medical Journal Editors (ICMJE) and is fully responsible for the content and editorial decisions.

Disclosure and conflicts of interest The authors received no funding for this manuscript and declare no conflict of interest. 
Open Access This article is distributed under the terms of the Creative Commons Attribution Noncommercial License which permits any noncommercial use, distribution, and reproduction in any medium, provided the original author(s) and source are credited.

\section{References}

1. Kalin, R., \& Stanton, M. S. (2005). Current clinical issues for MRI scanning of pacemaker and defibrillator patients. Pacing and Clinical Electrophysiology, 28(4), 326-328.

2. Harris, C. H. (2008). MRI procedure growth rate slows, study shows. Business \& Industry Digital Community Web site. http:// www.auntminnie.com/index.asp? sec=sup\&sub=bai\&pag=dis \& ItemId=81355. Accessed 15 September 2010.

3. Sutton, R., Kanal, E., Wilkoff, B. L., Bello, D., Luechinger, R., Jenniskens, I., et al. (2008). Safety of magnetic resonance imaging of patients with a new Medtronic EnRhythm MRI SureScan pacing system: clinical study design. Trials, 9, 68.

4. Sakakibara, Y., \& Mitsui, T. (1999). Concerns about sources of electromagnetic interference in patients with pacemakers. Japanese Heart Journal, 40(6), 737-743.

5. Levine, G. N., Gomes, A. S., Arai, A. E., Bluemke, D. A., Flamm, S. D., Kanal, E., et al. (2007). Safety of magnetic resonance imaging in patients with cardiovascular devices: an American Heart Association scientific statement from the Committee on Diagnostic and Interventional Cardiac Catheterization, Council on Clinical Cardiology, and the Council on Cardiovascular Radiology and Intervention: endorsed by the American College of Cardiology Foundation, the North American Society for Cardiac Imaging, and the Society for Cardiovascular Magnetic Resonance. Circulation, 116(24), 2878-2891.

6. Pohost, G. M., Blackwell, G. G., \& Shellock, F. G. (1992). Safety of patients with medical devices during application of magnetic resonance methods. Annals of the New York Academy of Sciences, 649, 302-312.

7. Gimbel, J. R., \& Kanal, E. (2004). Can patients with implantable pacemakers safely undergo magnetic resonance imaging? Journal of the American College of Cardiologists, 43(7), 1325-1327.

8. Shellock, F. G., \& Crues, J. V. I. I. I. (2002). MR Safety and the American College of Radiology White Paper. AJR. American Journal of Roentgenology, 178(6), 1349-1352.

9. Kanal, E., Borgstede, J. P., Barkovich, A. J., Bell, C., Bradley, W. G., Etheridge, S., et al. (2004). American College of Radiology White Paper on MR Safety: 2004 update and revisions. AJR. American Journal of Roentgenology, 182(5), 1111-1114.

10. Roguin, A., Schwitter, J., Vahlhaus, C., Lombardi, M., Brugada, J., Vardas, P., et al. (2008). Magnetic resonance imaging in individuals with cardiovascular implantable electronic devices. Europace, 10(3), 336-346.

11. Lauck, G., von Smekal, A., Wolke, S., et al. (1995). Effects of nuclear magnetic resonance imaging on cardiac pacemakers. Pacing and Clinical Electrophysiology, 18(8), 1549-1555.

12. Vahlhaus, C., Sommer, T., Lewalter, T., et al. (2001). Interference with cardiac pacemakers by magnetic resonance imaging: are there irreversible changes at 0.5 Tesla? Pacing and Clinical Electrophysiology, 24(4 Pt 1), 489-495.
13. Mollerus, M., Albin, G., Lipinski, M., \& Lucca, J. (2009). Ectopy in patients with permanent pacemakers and implantable cardioverter-defibrillators undergoing an MRI scan. Pacing and Clinical Electrophysiology, 32(3), 772-778.

14. Martin, E. T., Coman, J. A., Shellock, F. G., Pulling, C. C., Fair, R., \& Jenkins, K. (2004). Magnetic resonance imaging and cardiac pacemaker safety at 1.5-Tesla. Journal of the American College of Cardiologists, 43(7), 1315-1324.

15. Sommer, T., Naehle, C. P., Yang, A., Zeijlemaker, V., Hackenbroch, M., Schmiedel, A., et al. (2006). Strategy for safe performance of extrathoracic magnetic resonance imaging at 1.5 tesla in the presence of cardiac pacemakers in non-pacemaker-dependent patients: a prospective study with 115 examinations. Circulation, 114(12), $1285-1292$.

16. Luechinger, R., Duru, F., Zeijlemaker, V. A., Scheidegger, M. B., Boesiger, P., \& Candinas, R. (2002). Pacemaker reed switch behavior in $0.5,1.5$, and 3.0 Tesla magnetic resonance imaging units: are reed switches always closed in strong magnetic fields? Pacing and Clinical Electrophysiology, 25(10), $1419-1423$.

17. Sommer, T., Vahlhaus, C., Lauck, G., von Smekal, A., Reinke, M., Hofer, U., et al. (2000). MR imaging and cardiac pacemakers: in-vitro evaluation and in-vivo studies in 51 patients at $0.5 \mathrm{~T}$. Radiology, 215(3), 869-879.

18. Achenbach, S., Moshage, W., Diem, B., Bieberle, T., Schibgilla, V., \& Bachmann, K. (1997). Effects of magnetic resonance imaging on cardiac pacemakers and electrodes. American Heart Journal, 134(3), 467-473.

19. Luechinger, R., Zeijlemaker, V. A., Pedersen, E. M., Mortensen, P., Falk, E., Duru, F., et al. (2005). In vivo heating of pacemaker leads during magnetic resonance imaging. European Heart Journal, 26(4), 376-383. discussion 325-377.

20. Roguin, A., Zviman, M. M., Meininger, G. R., Rodrigues, E. R., Dickfeld, T. M., Bluemke, D. A., et al. (2004). Modern pacemaker and implantable cardioverter/defibrillator systems can be magnetic resonance imaging safe: in vitro and in vivo assessment of safety and function at 1.5 T. Circulation, 110(5), 475-482.

21. Mollerus, M., Albin, G., Lipinski, M., \& Lucca, J. (2008). Cardiac biomarkers in patients with permanent pacemakers and implantable cardioverter-defibrillators undergoing an MRI scan. Pacing and Clinical Electrophysiology, 31(10), 1241-1245.

22. Wilkoff, B. L., Bello, D., Taborsky, M., Vymazal, J., Kanal, E., Heuer, H., et al. (2010). Magnetic resonance imaging in patients with a pacemaker system designed for the magnetic resonance environment. Hear Rhythm, 8(1), 65-73.

23. Forleo, G. B., Santini, L., Della Rocca, D. G., Romano, V., Papavasileiou, L. P., Magliano, G., et al. (2010). Safety and efficacy of a new magnetic resonance imaging-compatible pacing system: early results of a prospective comparison with conventional dual-chamber implant outcomes. Hear Rhythm, 7(6), 750-754.

24. Gimbel, J. R. (2010). The safety of MRI scanning of pacemakers and ICDs: what are the critical elements of safe scanning? Ask me again at 10,000. Europace, 12(7), 915-917.

25. Faris, O. P., \& Shein, M. J. (2005). Government viewpoint: U.S. Food \& Drug Administration: pacemakers, ICDs and MRI. Pacing and Clinical Electrophysiology, 28(4), 268-269.

26. Gimbel, J. R. (2009). Unexpected asystole during 3 T magnetic resonance imaging of a pacemaker-dependent patient with a 'modern' pacemaker. Europace, 11, 1241-1242. 